\title{
Robotic equipment for deep-sea operation: Digital mock-up and assessment
}

\author{
E. Cavallo, R.C. Michelini, R.M. Molfino and R.P. Razzoli \\ Industrial Robot Design Research Group - University of Genova
}

\begin{abstract}
The paper presents a robotic arm, carried by a deep-see vehicle and purposely designed to perform precision handling for specialised maintenance tasks. The duties, up now performed by trained divers, are suitably defined, but remains rather compelling, due to the surroundings and with rising risk, as the depth increases. Resort to general-purpose, high-versatility robots happens to be unfair, as for sophistication requirements, and ineffective, as for duty bent; specialisation, the other hand, needs careful operation acknowledgement and proper task training. The considered problem deals with routine operations, and a purposely adapted contrivance is developed: the functional bent suggests to resort to a parallel kinematics manipulator. Design difficulties are the main drawbacks; the proposed solution, then, largely deals with digital mock-up and virtual testing assessment, for initially choosing the duty tailored fixture, thereafter for the life-cycle checks up to extreme deep-sea struggles.
\end{abstract}

Key Words: Integrated design, Digital mock-up, Instrumental robotics

\section{INTRODUCTION}

Underwater robotics has a long history, with, possibly, military-driven applications and, in many cases, looking after high-tech implementations, as particularly demanding tasks are sought. The situation is fast evolving, with the broader expansion of routine-applications, required to exploit the natural resources for mankind benefit everywhere they are placed. The preliminary undersea-surveying has generally been accomplished by the direct actions of 
frogmen or by remote-manipulation supervised from special vehicles by front-end operators. The unfriendly surroundings dramatically bounds the reachable work-areas and the duties that can be put in executions, without a raising in costs, not easily achieving balanced return on investments. This is suggesting a completely new approach in underwater robotics [1], [9], [3], [4] to look after duty-driven contrivances, properly goal-oriented.

The solution effectiveness depends on the ability of transferring well established technologies, so that low-cost devices could be developed to face the sub-set of adverse tasks as these pop out each time new surrounding are brought to work. At the moment, the off-shore structures for oil drawing out represent widely spread plants, requiring life-long inspection, regulation and maintaining operations, most of the time, strictly specialised, so that dutydriven fixtures could be devised to reach reliable efficiency. At dismissal, the wrecked plants become a non-bearable nuisance for the sea-life and the existing national bylaws require the safe and efficient restitution of previous conditions by dismantling and removing the old crocks.

The oil and gas industry is thus becoming an important purchaser of these diving-tools, and robotics founds out a promising application domain, on condition that transfer of the appropriate technology is suitably modulated to face actual tasks. In this paper, example topics on the transfer of parallel kinematics manipulators are recalled. The investigation has already dealt with the main conditioning facts [10], [11] hereafter, the procedure of linking structural architectures and functional properties is presented and appropriate digital mock-ups are developed, to supply virtual testing means, according to integrated design rules.

\section{PARALLEL KINEMATICS MANIPULATION}

Parallel kinematics manipulators are very little exploited, even when they might represent the most effective answer for a given requirement, due to the difficulties in trajectory tracking and path planning brought back by solving the backward kinematics. The hindrance is factually removed if proper CAD codes are available, so that the designer directly addresses the conditioning requirements, without a beforehand immersion in the peculiarities of closedloop mechanisms. In the following, only typical features of this integrated design process are summarised, sending back to the specialised literature for the pertinent details. For explanatory purposes, the study considers a typical six-degrees-of-freedom lay-out, Fig. 1, since it can be taken to represent a standard reference for the development process. The sequence of the topics is organised as follows: first, the qualitative description of the equipment properties is given; the design framework to be used for achieving proper 
functional performance is, then, presented, with the example mapping of the duty behavioural characteristics. Concluding comments provide further hints worked out from the example development.

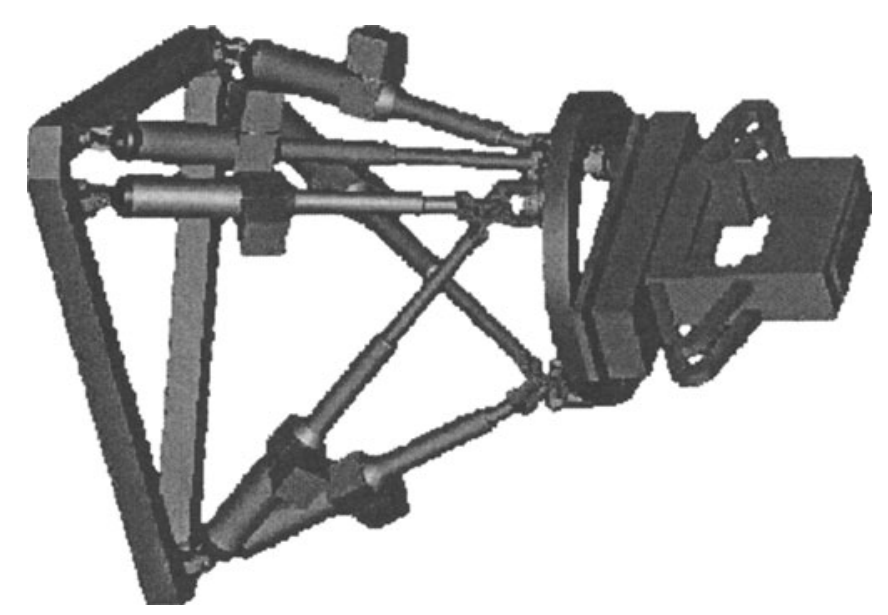

Figure 1. The 6 d.o.f. parallel platform lay-out

\subsection{Functional characterisation}

High stiffness and low inertial coupling are peculiar features of parallel mechanisms; work-space bounds and path singularities are main drawbacks. Thereafter, high force actuation along a properly bounded trajectory is considered typical work-cycle, to be performed with large acceleration and accuracy even if a simple open-loop position command is used. The example choice considers a six-legs Stewart platform, employed to accomplish the replacement of faulty electrovalves, placed in the control manifold of the $\mathrm{X}$-mas trees laying on the sea bottom of a gas drawing site. The task requests comparatively high forces and very accurate positioning; the correct analysis of the relationship between, on one side, the joint-space velocities and actuators forces and, the other side, the work-space velocities and effector forces (applied by the moving platform) needs reach properly balanced issues.

The investigation is carried on, referring to the forward kinematics of the mechanism; virtual assessments play a relevant role to work out quantitative figures of the performance for the different required engagements. The topic has been investigated in the past, and several authors [7], [6], have described the velocities and the force transmission characteristics by means of varying 
gain couplings, represented by the local Jacobian matrices, shown as hyperellipsoids around every task location of the work-space.

For design purposes, the six-dimensional input-output transformation of the chosen six-legs Stewart platform is split into two couples of sub-spaces, binding linear and angular displacements and, respectively, force and torque transmission. The manipulator behaviour, as a all, is highly non-linear, but the analysis makes possible the calculation of the locally linearised gains, so that the command can have before-hand setting and trimming, once the duty is selected.

\subsection{Characterising design framework}

The design framework, as previously stated, separately deals, splitting the manipulator Jacobian $\mathrm{J}$ in a translation and in a rotation part.

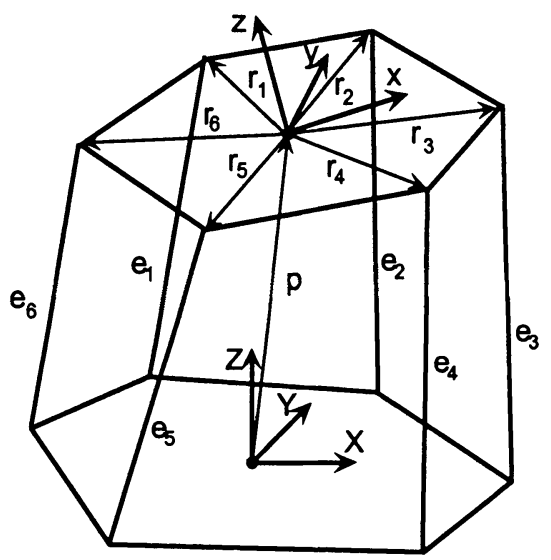

Figure 2. Geometry reference vectors

According to the notation explained in Fig. 2, the $6 \times 6$ Jacobian matrix of a Stewart like platform can be expressed as:

$$
\mathbf{J}=\left[\begin{array}{cccc}
\mathbf{r}_{1} \times \mathbf{e}_{1} & \mathbf{r}_{2} \times \mathbf{e}_{2} & \cdots & \mathbf{r}_{6} \times \mathbf{e}_{6} \\
\mathbf{e}_{1} & \mathbf{e}_{2} & \cdots & \mathbf{e}_{6}
\end{array}\right]^{T}
$$

It is well known that the relation between the input actuation and the output actions, force and torque, of the moving platform is locally expressed by the transposed Jacobian:

$$
\mathbf{F}=\mathbf{J}^{\mathbf{T}} \mathbf{f}
$$


where: the vector $\mathbf{f}=\left(f_{1}, \ldots, f_{6}\right)^{T}$ represents the actuation force and the vector $\mathbf{F}=\left(f_{o}^{T}, m_{0}{ }^{T}\right)^{T}$ represents output force/torque exerted by the moving platform.

In order to simplify the mechanism analysis, is convenient to consider separately the output force and torque; such a formulation is very useful for the mechanism design steps, when the attention is focused on the net output force either torque components, as, depending on task requests, one has to evaluate the force the robot can exert without generating any torque, or vice versa.

On these premises, the generic expression (2) is decomposed into:

$$
\left(\begin{array}{l}
f_{0} \\
m_{0}
\end{array}\right)=\left[\begin{array}{c}
J_{t}^{T} \\
J_{r}^{T}
\end{array}\right] f
$$

where: the two $3 \times 6$ matrices $J_{t}^{T}$ and $J_{r}^{T}$ express the relations between the actuation input and the effector net force either respectively net torque.

Applying the definition of the velocity and force/torque ellipsoids to the two simplified Jacobian matrices [8], we obtain that the output force exerted by the moving platform form an ellipsoid, whose axes lie on the direction of the eigenvectors of the matrix $\left[J_{t}^{T}\right]$ and their magnitudes are the square root of the matrix eigenvalues. Similarly, it is possible to express the net torque ellipsoid making use of the $\left[J_{r} J_{r}^{T}\right]$ matrix.

The analysis has to be repeated for every point of the work-space. The robot behaviour is, thereafter, is fully specified evaluating, for all authorised manipulator configurations corresponding to chosen tip position and attitude, the exerted:

\# force, applied without generating moment (net force ellipsoid); or: \# torque, transmitted without generating effort (net torque ellipsoid).

Remembering that the evaluation of the amplification between internal and external velocity is in the same way linked to the inverse of the two above matrices, the inquiry is easily extended to the linear and rotational velocity ellipsoids; moving accordingly, the velocity ellipsoids are evaluated distinguishing linear from rotational displacement missions:

\# linear local path of the platform centroid (net displacement ellipsoid); \# angular local attitude around the platform centroid (net angular ellipsoid). In this case, it is useful to remember that, due to the definition, the principal axes of the velocity and of the force ellipsoids coincide, and the length of the axes are in inverse proportions. In fact, the force ellipsoid, defined by $\left[\mathrm{JJ}^{\mathrm{T}}\right]$, and the velocity ellipsoid, given by $\left[\mathrm{JJ}^{\mathrm{T}}\right]^{-1}$, have the same eigenvectors and the eigenvalues are in reciprocal proportion.

The reciprocity between velocity and force ellipsoids has an important consequence: the configurations in which the force transmission reaches the 
maximum value is the worst for the control of the manipulator, when related to the velocity transmission. The final setting of the manipulator, thereafter, can actually be achieved once the work-space analysis is completed and the appropriate parameter (size, geometry, actuation gains ...) balancing ensures the task execution.

This kind of investigation has been accomplished. For example purposes, a customisable Stewart-like 6 d.o.f. parallel platform is considered and the repeated solutions for every configurations are numerically obtained. The set of net force ellipsoids, Fig. 3, of net torque ellipsoids, Fig. 4, of net displacement ellipsoids, Fig. 5, net angular ellipsoids, Fig. 6, show the local manipulator behaviours and allow to foresee the functional characteristics of the mechanism, when characterised on merely input-output actuation gains, either, velocity ratios.

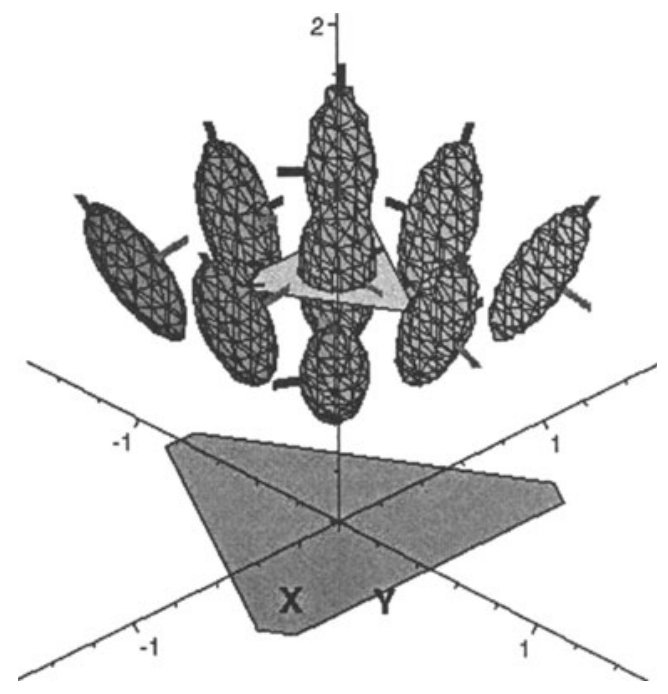

Figure 3. Net force ellipsoids for 9 different workspace locations

The parallel kinematics architectures, as previously recalled, are mainly used for comparatively large (generalised) force transmission, along highaccuracy trajectories; in these situations, they grant built-in propensity and can avoid the addition of sensing elements on the effector and feedback loop, always critical for under-sea applications. The sketched transform matrices description provides an effective aid for tuning the driving force actuation in dependence of the desired work-cycles and with balanced concern with the tracked trajectory accuracy. The actually selected robot comes out removing 
the fear of addressing unconventional mechanical structures, as the useful design information are made available as virtual benchmark .

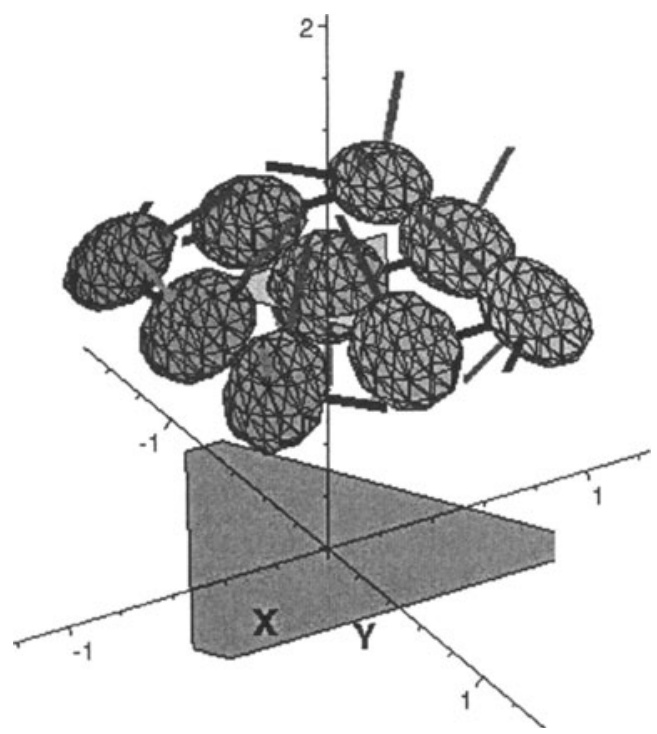

Figure 4. Net torque ellipsoids for 9 different workspace locations

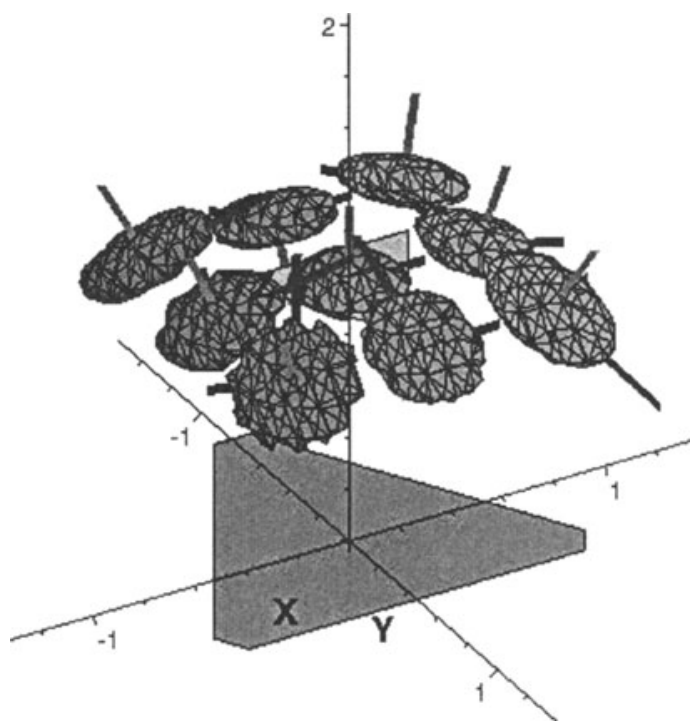

Figure 5. Net linear velocity ellipsoids at the same work-space locations 
The sample issues given in the paper aims at providing explanatory hints of the design process in view of recurrently trimming force and displacement transmission. The overall project carries on the investigation by kinetic and dynamic simulations on multi-body models and by FEM analysis, to enrich the knowledge about the machine behaviour. The preliminary investigation ends by setting the robot structural lay-out and by selecting the set of subassemblies assuring the sought performance (in this case, for instance, the functional and structural optimisation of the top double spherical and of the bottom universal joints is sought).

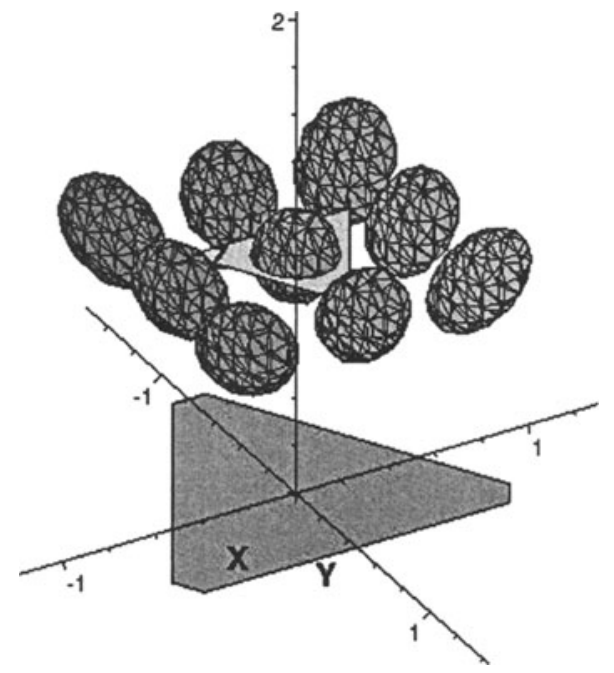

Figure 6. Net rotational velocity ellipsoids at the same work-space locations

The complete set of data on the mechanism structure permits the designer to define, since the first steps of the process, the better choices to achieve the desired performance. For deep-sea applications, effective virtual prototyping [5] and testing bear particular relevance, as the experimentation on real settings is dangerous and expensive. Happily enough, nowadays, the utilisation of software packages available for geometric, structural, cinematic and control simulations gives not only an added value to the quality of products, but also an indispensable contribution to achieve significant lifecycle results, with friendly graphic rendering. This is the way, we intend "virtual reality", that is the description of every peculiar feature of the object by means of the appropriate model and the analysis to extract characteristics and information about the system behaviour under actual running conditions. The opportunity was largely exploited along with the project development, and the principal results where discussed in previous papers; here details on the joint- to the work-space transforms are recalled due to their effects on the 
diffusion of parallel kinematics mechanisms, even when these happen to be best choice.

\section{CONCLUDING COMMENTS}

The instrumental robots are properly selected due to their functional-bent. To that purport, increasing attention has recently been focussed on parallel kinematics manipulator, due to their ability of high accuracy path tracking, with open loop command. The spreading out of the technology founds great hindrance, because of difficulties in solving the forward kinematics. In the paper, a procedure leading to an effective choice of a typical 6 d.o.f. parallel manipulator is recalled, giving the basic reference, based on standard CAD tools, for assessing the work-space cinematic and dynamic performance.

The analysis is grounded on numerical computations, where the Jacobian matrices are properly used; these relate a six dimensional input vector to a six dimensional output vector, making possible the full work-space analysis in terms of generalised force, either, generalised displacement transmission matrices. For practical purposes, the investigation is better tackled, splitting the analysis as previously recalled; in fact, actual duties suitably distinguish force from torque tasks (and linear from angular displacement tasks). This makes possible very effective restitution into tree-dimensions, suggesting the setting of the open-loop gains according to functional-driven requests.

The deep-sea development resulted in a very effective setting; the special purpose robot, based on the chosen Stewart-like 6 d.o.f. parallel platform, makes possible to move heavy objects with high accelerations and accuracy, achieving the expected performance with a simple and rugged device, not requiring sophisticated sensors and feed-backs. According to this study, the peculiar problem of plugging-in electrovalves on submarine control panels is efficiently solved using a task oriented parallel architecture, able to exert due force to execute the insertion and extraction operations.

Summing up, the proposal did resort to a comparatively unconventional architecture; to assess the benefits, virtual prototyping and testing [2] are used to look after the operation reliability of the device, by duty-driven assignments, to lower development investment and rise life-cycle effectiveness. In the future, the replacing of divers will become appropriate technology, whether the anthropocentric view is dropped and rigs suitably designed following engineering rules are procured. This means to accomplish feasibility studies, to compare competing solutions, to acknowledge on-process performance, to test recovery sequences, etc. before starting the manufacturing of any special artefacts; and "virtual reality" testing is basic tool to this scope. 


\section{REFERENCES}

[1] Acaccia, G.M., Callegari, M., Michelini, R.C., Molfino, R.M., Razzoli, R.P.: "Underwater robotics: example survey and suggestions for effective devices", 4th. ECPD Intl. Conf. Advanced Robotics, Intelligent Automation \& Active Systems, Moscow, Aug. 24-26, 1998, pp. 409-416.

[2] Acaccia, G.M., Cavallo, E., Garofalo, E., Michelini, R.C., Molfino, R.M., Callegari, M.: "Remote manipulator for deep-see operations: animation and virtual reality assessment", in Proc. 2nd Workshop on Harbour, Maritime \& Logistics Modelling and Simulation (HMS99), 16-18 September 1999, Genova, Italy, pp.57-62, ISBN 156555 1753.

[3] Batlle, J., Fuertes, J.M., Marti, J., Pacheco, Ll. and Meléndez, J.: “Telemanipulated arms for underwater applications", Proceedings, Seventh Intl. Conf. on Advanced Robotics ICAR '95, Sant Feliu de Guixols, Catalonia, Spain, Vol. 1, pp. 267-272, 1995.

[4] Bruzzone, L.E., Cavallo, E.M., Michelini, R.C., Molfino, R.M,. Razzoli, R.P.: "The design of a robotic equipment for deep sea maintenance operation", Proc. ASME Engineering Systems Design and Analysis Conference 2000, Montreaux, Switzerland, July 10-13, 2000.

[5] Callegari, M., Cavallo, E., Garofalo, E., Michelini, R.C., Molfino, R.M., Razzoli, R.P.: "The design of diving robots: set-up assessment by virtual mock-ups", Proc. of the XI ADM Intl. Conf. on Design Tools and Methods in Industrial Engineering, Palermo 8-12 Dic. 1999, vol. C - pp. 147-154.

[6] Chiu, S.: "Kinematic characterization of manipulators: an approach to defining optimality", IEEE Int. Conf. Robotics and Automation (1988), pp. 828-833

[7] Kosuge, K., Okuda, M., Fukida, T., Koduka, T., Mizuno, T.: "Input/output force analysis of Stewart platform type of manipulators", Proc. Of 1993 IEEE/RSJ Intl. Conf. On Intelligent Robots and Systems, Yokohama, Japan, July 26-30, 1993, pp. 16661673.

[8] Ma, O., Angeles, J.: "Architecture singularities of platform manipulators", Proceedings of the 1991 IEEE Intl. Conf. On Robotics and Automation, Sacramento, California, pp. 1542-1547.

[9] Maddalena, D., Prendin, W., and Terribile, A.: "Underwater Telerobotics reaches the field”, Proceedings, 27th ISIR Robotics towards 2000, Milan, Italy, pp. 167-172, 1996.

[10] Michelini, R.C., Molfino, R.M., Razzoli, R.P., Rio, A., Truffelli, F.: “A parallel kinematics robotic arm for deep sea operations", Proc. Intl. Workshop on Parallel Kinematics Machines (PKM99), Milano, Italy, Nov. 30th, 1999, pp. 243-250.

[11] Sayers, C.P., Yoerger, D.R., Paul, R.P., Lisievich, J.S.: "A manipulator work package for teleoperation from unmanned untethered vehicles: current feasibility and future applications", Proceedings, 6th IARP Workshop on Underwater Robotics, Toulon-La Seyne, France, 1996. 